\title{
Expression and prognosis analyses of PEA3 subfamily in head and neck squamous cell carcinoma via integrated bioinformatics
}

mei wei

Tianjin First Central Hospital

wei wang ( $\square$ Wwei1106@hotmail.com )

Tianjin First Central Hospital https://orcid.org/0000-0002-0217-3074

guangjian ni

Tianjin University

\section{Research}

Keywords: Head and neck squamous cell carcinoma, PEA3 subfamily, Prognosis.

Posted Date: January 25th, 2022

DOI: https://doi.org/10.21203/rs.3.rs-402839/v2

License: (c) (i) This work is licensed under a Creative Commons Attribution 4.0 International License.

Read Full License 


\section{Abstract \\ Backgroud:}

The PEA3 subfamily belongs to the first category of the ETS family, and it consists of three family members, namely, ETV1, ETV4, and ETV5. These members were implicated in the progression of many cancers. But their prognostic roles in head and neck squamous cell carcinoma (HNSCC) remain to be characterized.

\section{Methods}

Data from multiple open databases were used to analyze the expression, regulation, mutations, immune infiltration, and functional networks of the PEA3 subfamily in patients with HNSCC.

\section{Results}

The results demonstrated that these patients showed downregulated ETV1 and upregulated ETV4/5 compared with healthy subjects. Furthermore, high expression of ETV4/5 and low expression of ETV1 were linked to the shortened overall survival in patients with HNSCC. The immune infiltration in B cells stimulated the prognosis, and it may have a prognostic medical significance linked to the PEA3 subfamily in HNSCC. These results showed that data mining efficiently revealed the expression of the PEA3 subfamily in HNSCC, but this study requires further large-scale genomic and basic research.

\section{Conclusions}

This study provided new insight into the prognostic roles of PEA3 subfamily in HNSCC with potential mechanistic values.

\section{Introduction}

Head and neck squamous cell carcinomas (HNSCCs) are the seventh most common cancer worldwide, occurring in more than half a million new patients every year, which have seriously endangered the lives and health of the population ${ }^{[1,2]}$. The 5-year overall survival rate (OS) of HNSCC patients is poor, and the survival rate of patients with local recurrence and metastasis is even lower ${ }^{[3,4]}$. Therefore, in order to better prognosis patients with head and neck squamous cell carcinoma, it is necessary to determine new indicators for prognostic evaluation and targeted therapy of HNSCC.

The E26 transformation specificity (ETS) family comprises the most prominent signal-dependent transcription factor families, consisting of 28 protein-coding genes in the human genome ${ }^{[5]}$. ETS transcription factors participate in tumorigenesis and development by regulating various biological 
processes, such as cell proliferation, migration, apoptosis, aging, angiogenesis, and stem-cell development ${ }^{[6]}$. They are divided into several different subfamilies based on the ETS domain's high amino acid conservation and the subgroup-specific amino acid sequence ${ }^{[7]}$. The PEA3 subfamily belongs to the first category of the ETS family, and it consists of three family members, namely, ETV1, ETV4, and ETV5, which were implicated in the progression of many cancers ${ }^{[8]}$. ETV1 is often deregulated in prostate cancer ${ }^{[9]}$ and expressed explicitly in most gastrointestinal stromal tumors ${ }^{[10]}$. The ETV4 transcription factor was frequently activated in gastric cancer ${ }^{[11]}$, lung cancer ${ }^{[12]}$, hepatocellular carcinoma ${ }^{[13]}$, and ovarian cancer. ETV5 was implicated in endometrial cancer progression ${ }^{[14]}$, thyroid cancer $^{[15]}$, and ovarian cancer ${ }^{[16]}$.

Given the extensive carcinogenic effects of the PEA3 subfamily, ETV1, ETV4, and ETV5 have been proposed as prognostic markers for patients with cancer ${ }^{[17]}$. In our study, the mutation signatures and expression levels of the PEA3 subfamily in HNSCC datasets were explored through integrated bioinformatics analysis to provide new insights into their potential functions, molecular mechanisms, and prognostic significance.

\section{Materials And Methods}

\section{Oncomine database}

The expression data of PEA3 mRNAs in HNSCC and standard samples were obtained from Oncomine (https://www.oncomine.org/ ) [18], a database that currently includes 715 gene expression datasets and 86,733 samples, and $p<0.05$ was used as the cut-off criterion.

\section{Gene Expression Profiling Interactive Analysis (GEPIA) dataset and TNM Database}

GEPIA ( http://gepia.cancer-pku.cn/ ) ${ }^{[19]}$ is a newly created interactive online database that allows users to find RNA-seq expression data or samples based on Genotype-Tissue Expression projects (GTEx) and The Cancer Genome Atlas (TCGA). The database is based on a criterion processing pipeline and offers customizable functions, such as profiling on pathological stages, cancer types, differential expression analysis, survival analysis, correlation analysis, and similar gene detection. TNMplot ( https://www.tnmplot.com// ${ }^{[20]}$ is an integrated database that uses available transcriptome-level datasets to compare typical tumors and metastatic data across all genes in real-time. In our study, this database was used to compare the expression of the PEA3 subfamily in normal and tumor tissues.

\section{UALCAN analysis}

UALCAN (http://ualcan.path.uab.edu) ${ }^{[21]}$ was used to analyze, integrate and discover transcriptomic cancer data and for in-depth analyses of TCGA gene expression information. The website could perform in-silico analysis of potential candidate genes of interest to assess the expression in various subgroups, such as age, gender, race, and grade. 


\section{Kaplan-Meier plotter}

Kaplan-Meier (K-M) plotter (http://kmplot.com/analysis/) ${ }^{[22]}$ was used to analyze the prognostic significance of the mRNA expression of different PEA3 subfamilies in HNSCC. The patients were divided into low- and high-expression groups in accordance with each PEA3 subfamily's median values and assessed using $\mathrm{K}-\mathrm{M}$ survival plot.

\section{cBioPortal and Genemania database}

The cBioPortal portal (http://cbioportal.org) ${ }^{[23]}$ encompassing datasets of 245 cancer studies was used to analyze PEA3 subfamily mutations. The GeneMANIA database( https://genemania.org/ ) ${ }^{[24]}$ is an online database used for searching known protein interaction relationships. It was used in the present study to explore the interaction network of the PEA3 subfamily, and the co-expressed genes were analyzed. GO (Gene Ontology) and KEGG (Kyoto Encyclopedia of Genes and Genomes) function analyses of the PEA3 subfamily and the co-expressed genes were performed on R software.

\section{Tumor Immune Estimation Resource (TIMER) Analysis}

TIMER (https://cistrome.shinyapps.io/timer/) ${ }^{[25]}$ is a comprehensive asset used to systematically evaluate tumor infiltration in CD8+ T cells, B cells, CD4+ T cells, macrophages, neutrophils, and dendritic cells and their correlation with the expression of the PEA3 subfamily in HNSCC. The survival module was used to determine immune infiltrates and the PEA3 subfamily to obtain the survival differences, in which $p<0.05$ was considered statistically significant.

\section{Results}

\section{Expression of PEA3 family members in HNSCC}

The mRNA and protein expression of the PEA3 subfamily was investigated using the ONCOMINE and GEPIA datasets. As shown in Fig. 1A and 1B, the expression of ETV1 was higher in the normal tissues than in the cancer tissues. The expression of ETV4 and ETV5, on the contrary, was higher in the cancer tissues. The TNM plot dataset was also used to compare the PEA3 expression amongst cancer and normal tissues. The results indicated that ETV4/5 was significantly higher in HNSCC tissues than in normal tissues ( $p<0.05$, Fig. 1C).

\section{Clinical subgroup analysis}

A subgroup analysis of multiple clinical-pathological features was conducted using the TCGA database. Subgroup analysis by age, metastasis, status, gender subgroup, and cancer stages indicated that the transcriptional levels of ETV1 were lower in patients with HNSCC than in healthy individuals. Besides, subgroup analysis by age, cancer stages, metastasis status, HPV status, gender subgroup, and tumor grade demonstrated that the transcriptional levels of ETV4 and ETV5 were significantly higher in patients with HNSCC across all subgroups (Fig. 2). 


\section{Prognostic analysis}

The prognostic significance, which was obtained from publicly available online datasets, of the PEA3 subfamily, was explored in patients with HNSCC. As shown in Fig. 3, the increased expression of ETV1 $(\mathrm{HR}=0.73,95 \% \mathrm{Cl}: 0.56-0.96, p=0.021)$ was related to prolonged OS. The low expression levels of ETV4 $(H R=1.5,95 \% \mathrm{Cl}: 1.14-1.96, p=0.0031)$ and ETV5 (HR $=1.51,95 \% \mathrm{Cl}: 1.15-1.99, \mathrm{p}=0.0026)$ were also related to prolonged OS. These results suggested that the levels of PEA3 may play an important role in HNSCC prognosis.

\section{Predicted functions and pathways of PEA3 in HNSCC}

The mutation patterns and functional networks of PEA3 were analyzed using cBioPortal and GeneMANIA further to explore the biological role of PEA3 in HNSCC. Amongst the 827 HNSCC tumor samples sequenced, genetic alterations were detected in 132 samples, with a mutation rate of $16 \%$. ETV5 was ranked as the most mutated gene, with a mutation rate of $16 \%$. The network for PEA3 and 22 most frequently altered neighboring genes was also shown (Fig. 4).

The functions of PEA3 and these 22 genes were analyzed using GO and KEGG enrichment (Fig. 5). GO analysis indicated that the changes in biological processes included aging, positive regulation of hair cycle, extracellular matrix disassembly, replicative senescence, and negative regulation of metallopeptidase activity. The molecular function was mainly enriched in metalloendopeptidase inhibitor activity, transcription coactivator binding, mitogen-activated protein kinase binding, protein serine/threonine kinase activity, and transcription cofactor binding. Changes in cell components were primarily enriched in secretory granule lumen, cytoplasmic vesicle lumen, vesicle lumen, protein-lipid complex, and specific granule lumen. According to KEGG analysis results, pathway enrichment analysis was mainly enriched in transcriptional misregulation in cancer, prostate cancer, cellular senescence, cholesterol metabolism, VEGF signaling pathway, MAPK signaling pathway, and FoxO signaling pathway.

\section{Immune infiltrates in correlation with PEA3 in HNSCC}

A statistically significant correlation was found between PEA3 expression in HNSCC and immune infiltrates $(p<0.05$, Fig. 6$)$. The expression levels of ETV1 and ETV5 were positively correlated with the immune infiltration levels in HNSCC, HNSCC-HPV-pos, and HNSCC-HPV-neg, whereas that of ETV4 was negatively correlated. We hypothesize that infiltration of immune cells significantly affect prognosis. Therefore, we explored the difference in cumulative survival between HNSCC, HNSCC-HPV-pos, and HNSCC-HPV-neg. The results showed that B cells, CD8+ T cells, and neutrophils of immune infiltrates statistically significant to PEA3 subfamily levels in HNSCC-HPV-pos subgroup, indicating that these immune cell infiltrations significantly affect prognosis. (Fig. 7).

\section{Discussion}


In this study, we explored the expression levels and mutational landscape of various PEA3 subfamilies in multiple HNSCC datasets and to elucidate their exact function in HNSCC. The results showed that the expression levels of specific PEA3 subfamilies were correlated with prognosis and immune infiltration.

A growing number of studies have shown that the PEA3 subfamily promotes cell migration and invasion, thereby contributing to tumor progression and metastasis. Several studies have reported that the expression of ETV1 was important in muscle organ and cerebellar circuit development ${ }^{[26,27]}$. Lunardi et al. ${ }^{[28]}$ demonstrated that ETV1 was a negative regulator of checkpoint kinase 1 (CHK1) in prostate cancer. ETV1 overexpression could inhibit CHK1, leading to the accumulation of DNA damage and the promotion of invasive tumorigenesis. MMPs are common genes related to cell migration and invasion. Many studies have reported that ETV1 could stabilize $\beta$-catenin and directly bind to MMP1/7, resulting in increased accumulation of $\beta$-catenin and MMP1/7 and induced migration and invasion of prostate cancer cells ${ }^{[9,29,30]}$. A recent study has shown that the expression of ETV4 was activated by PI3K/Akt signaling in clear-cell RCC and ETV4 promoted cell migration by directly binding to its downstream promoter FOSL $1^{[31]}$. Moreover, in advanced prostate cancer, ETV4 has been reported to be overexpressed under the combinatorial activation of the PI3-kinase and RAS signaling pathways, indicating that ETV4 may represent a valuable therapeutic target in metastatic prostate cancer ${ }^{[32]}$. ETV5 was a target gene of the Src family kinase, and it promoted female germline stem cells ${ }^{[33]}$. Besides, ETV5 has been thought to be an obesity-related gene that was important for regulating energy balance and metabolism, including insulin secretion and glucocorticoid circulation ${ }^{[34,35]}$. Another study has also found that ETV5 acted as a transcription factor related to papillary thyroid cancer cell growth by targeting Twist 1 to trigger the EMT process $[17,36]$.

The present study showed that ETV4 and ETV5 were higher in the cancer tissues than in the normal tissues. High ETV4 and ETV5 were also found in patients with HNSCC, which was correlated with a poor prognosis. Contradictory to previous studies, this study revealed that ETV1 was lowly expressed in HNSCC tissues, and a low level of ETV1 could lead to poor prognosis, thereby warranting further exploration of its functions. The function and pathways of the PEA3 subfamily and their frequently altered neighboring genes were significantly enriched in aging, extracellular matrix disassembly, transcription coactivator binding, and other cell functions. KEGG analyses showed enrichment in the VEGF signaling pathway, MAPK signaling pathway, cellular senescence, and transcriptional misregulation in cancer. The MAPK signaling pathway is a crucial player in the initiation and progression of many cancers.

The role of the PEA3 subfamily and tumor-infiltrating immune cells were also explored. In the HNSCCHPV-pos group, a significantly increased infiltration of B cells, CD8+ T cells, and neutrophils were found $(p<0.05)$, which was sufficiently related to the expression of the PEA3 subfamily. This finding indicated that immune cells might have a significant effect on HNSCC prognosis. Therefore, the potential role of the PEA3 subfamily must be investigated further.

This study aimed to investigate the clinical value of the PEA3 subfamily in HNSCC and the molecular mechanism from big data. The findings may offer new perspectives in future research and clinical 
applications for patients with HNSCC. However, this study had several limitations. All the data analyzed were retrieved from online databases. Precise clinical information was not provided. Thus, the findings must be validated by further studies. Finally, additional research attention should be paid to the specific functions and mechanisms of the action of the PEA3 subfamily in HNSCC.

\section{Abbreviations}

$\mathrm{Cl}$

Confidential intervals

GEO

Gene Expression Omnibus

HR

Hazard ratio

GO

Gene Ontology

KEGG

Kyoto Encyclopedia of Genes and Genomes

OS

Overall survival

TIMER

Tumor Immune Estimation Resource

GEPIA

Gene Expression Profiling Interactive Analysis Platform

HNSCC

Head and Neck Squamous Cell Carcinoma.

\section{Declarations}

\section{Acknowledgements}

Not applicable.

\section{Funding}

This study was supported by the Key Clinical Discipline of Tianjin; National Natural Science Foundation of China (81971698); Science and Technology Project of Tianjin Municipal Health Commission (KJ20136). Science and Technology Project of Tianjin Municipal Health Commission (ZC20010).

\section{Authors' contributions}

Mei Wei carried out data analysis and drafted the manuscript; Wei Wang 
and Guangjian Ni participated in study design and data collection. All authors read and approved the final manuscript.

\section{Availability of data and materials}

The online databases used in the study:

Oncomine dataset: https://www.oncomine.org/

GEPIA dataset : http://gepia.cancer-pku.cn/

TNMplot dataset : https://www.tnmplot.com/

UALCAN: http://ualcan.path.uab.edu

Kaplan-Meier plotter: http://kmplot.com/analysis/

cBioPortal portal database: http://cbioportal.org

GeneMANIA database: https://genemania.org/

TIMER database: https://cistrome.shinyapps.io/timer/

All data generated or analysed during this study are included in this published article.

\section{Ethics approval and consent to participate}

This article does not contain any studies with human participants or animals performed by any of the authors, therefore no ethic approval or consent is required.

\section{Consent for publication}

Not applicable.

\section{Competing interests}

All authors declare no conflict of interest in this study.

\section{Author details}

1. Academy of Medical Engineering and Translational Medicine, Tianjin Key Laboratory of Brain Science and Neural Engineering, Tianjin University, Tianjin, China. 2. Laboratory of Neural Engineering and Rehabilitation, Department of Biomedical Engineering, College of Precision Instruments and Optoelectronics Engineering, Tianjin University, Tianjin, China. 3. Department of Otorhinolaryngology Head and Neck Surgery, Tianjin First Central Hospital, 300192 Tianjin, China 
4. Institute of Otolaryngology of Tianjin, China. 5. Key Laboratory of Auditory Speech and Balance Medicine, Tianjin, China 6. Key Clinical Discipline of Tianjin (Otolaryngology), China. 7. Otolaryngology Clinical Quality Control Centre, Tianjin, China.

\section{References}

1. Ferlay J, Shin HR, Bray F, et al. Estimates of worldwide burden of cancer in 2008: GLOBOCAN 2008 [J]. Int J Cancer. 2010;127(12):2893-917.

2. Arthur AE, Goss AM, Demark-Wahnefried W, et al. Higher carbohydrate intake is associated with increased risk of all-cause and disease-specific mortality in head and neck cancer patients: results from a prospective cohort study [J]. Int J Cancer. 2018;143(5):1105-13.

3. Simard EP, Ward EM, Siegel R, et al. Cancers with increasing incidence trends in the United States: 1999 through 2008 [J]. CA Cancer J Clin. 2012;62(2):118-28.

4. Chin D, Boyle GM, Williams RM, et al. Novel markers for poor prognosis in head and neck cancer [J]. Int J Cancer. 2005;113(5):789-97.

5. Oh S, Shin S, Janknecht R. ETV1, 4 and 5: an oncogenic subfamily of ETS transcription factors [J]. Biochim Biophys Acta. 2012;1826(1):1-12.

6. Sementchenko VI, Watson DK. Ets target genes: past, present and future [J]. Oncogene. 2000;19(55):6533-48.

7. de Launoit Y, Chotteau-Lelievre A, Beaudoin C, et al. The PEA3 group of ETS-related transcription factors. Role in breast cancer metastasis [J]. Adv Exp Med Biol. 2000;480:107-16.

8. Wang B, Krall EB, Aguirre AJ, et al. ATXN1L, CIC, and ETS Transcription Factors Modulate Sensitivity to MAPK Pathway Inhibition [J]. Cell Rep. 2017;18(6):1543-57.

9. Morsalin S, Yang C, Fang J, et al. Molecular Mechanism of $\beta$-Catenin Signaling Pathway Inactivation in ETV1-Positive Prostate Cancers [J]. J Pharm Sci Pharmacol. 2015;2(3):208-16.

10. Eid W, Abdel-Rehim W. Genome-wide analysis of ETV1 targets: Insights into the role of ETV1 in tumor progression [J]. J Cell Biochem. 2019;120(6):8983-91.

11. Zhang X, Wang Y, Liu X, et al. KIF2A promotes the progression via AKT signaling pathway and is upregulated by transcription factor ETV4 in human gastric cancer [J]. Biomed Pharmacother, 2020, 125(109840.

12. Cheng T, Zhang Z, Cheng Y, et al. ETV4 promotes proliferation and invasion of lung adenocarcinoma by transcriptionally upregulating MSI2 [J]. Biochem Biophys Res Commun. 2019;516(1):278-84.

13. Yang QX, Zhong S, He L, et al. PBK overexpression promotes metastasis of hepatocellular carcinoma via activating ETV4-uPAR signaling pathway [J]. Cancer Lett. 2019;452:90-102.

14. Pedrola N, Devis L, Llauradó M, et al. Nidogen 1 and Nuclear Protein 1: novel targets of ETV5 transcription factor involved in endometrial cancer invasion [J]. Clin Exp Metastasis. 2015;32(5):467-78. 
15. Meng D, Li Z, Ma X, et al. ETV5 overexpression contributes to tumor growth and progression of thyroid cancer through PIK3CA [J]. Life Sci, 2020, 253(117693.

16. Llauradó M, Majem B, Castellví J, et al. Analysis of gene expression regulated by the ETV5 transcription factor in OV90 ovarian cancer cells identifies FOXM1 overexpression in ovarian cancer [J]. Mol Cancer Res. 2012;10(7):914-24.

17. Qi T, Qu Q, Li G, et al. Function and regulation of the PEA3 subfamily of ETS transcription factors in cancer [J]. Am J Cancer Res. 2020;10(10):3083-105.

18. Rhodes DR, Kalyana-Sundaram S, Mahavisno V, et al. Oncomine 3.0: genes, pathways, and networks in a collection of 18,000 cancer gene expression profiles [J]. Neoplasia. 2007;9(2):166-80.

19. Tang Z, Li C, Kang B, et al. GEPIA: a web server for cancer and normal gene expression profiling and interactive analyses [J]. Nucleic Acids Res. 2017;45(W1):W98-w102.

20. Bartha Á, Győrffy B. TNMplot.com: a web tool for the comparison of gene expression in normal, tumor and metastatic tissues [J]. bioRxiv, 2020, 2020.2011.2010.376228.

21. Chandrashekar DS, Bashel B, Balasubramanya SAH, et al. UALCAN: A Portal for Facilitating Tumor Subgroup Gene Expression and Survival Analyses [J]. Neoplasia. 2017;19(8):649-58.

22. Nagy Á, Lánczky A, Menyhárt 0 , et al. Validation of miRNA prognostic power in hepatocellular carcinoma using expression data of independent datasets [J]. Sci Rep. 2018;8(1):9227.

23. Cerami E, Gao J, Dogrusoz U, et al. The cBio cancer genomics portal: an open platform for exploring multidimensional cancer genomics data [J]. Cancer Discov. 2012;2(5):401-4.

24. Franz M, Rodriguez H, Lopes C, et al. GeneMANIA update 2018 [J]. Nucleic Acids Res. 2018;46(W1):W60-w64.

25. Li T, Fan J, Wang B, et al. TIMER: A Web Server for Comprehensive Analysis of Tumor-Infiltrating Immune Cells [J]. Cancer Res. 2017;77(21):e108-10.

26. Tenney AP, Livet J, Belton T, et al. Etv1 Controls the Establishment of Non-overlapping Motor Innervation of Neighboring Facial Muscles during Development [J]. Cell Rep. 2019;29(2):43752.e434.

27. Yang JJ, Bertolesi GE, Hehr CL, et al. Lhx2/9 and Etv1 Transcription Factors have Complementary roles in Regulating the Expression of Guidance Genes slit1 and sema3a [J]. Neuroscience. 2020;434:66-82.

28. Lunardi A, Varmeh S, Chen M, et al. Suppression of CHK1 by ETS Family Members Promotes DNA Damage Response Bypass and Tumorigenesis [J]. Cancer Discov. 2015;5(5):550-63.

29. Shin S, Oh S, An S, et al. ETS variant 1 regulates matrix metalloproteinase-7 transcription in LNCaP prostate cancer cells [J]. Oncol Rep. 2013;29(1):306-14.

30. Oh S, Shin S, Lightfoot SA, et al. 14-3-3 proteins modulate the ETS transcription factor ETV1 in prostate cancer [J]. Cancer Res. 2013;73(16):5110-9.

31. Xu L, Hu H, Zheng LS, et al. ETV4 is a theranostic target in clear cell renal cell carcinoma that promotes metastasis by activating the pro-metastatic gene FOSL1 in a PI3K-AKT dependent manner 
[J]. Cancer Lett. 2020;482:74-89.

32. Aytes A, Mitrofanova A, Kinkade CW, et al. ETV4 promotes metastasis in response to activation of $\mathrm{PI} 3-k i n a s e$ and Ras signaling in a mouse model of advanced prostate cancer [J]. Proceedings of the National Academy of Sciences of the United States of America, 2013, 110(37).

33. Zhang X, Wei R, Sun Y, et al. AKT3 Is a Pivotal Molecule of Cadherin-22 and GDNF Family Receptora1 Signal Pathways Regulating Self-Renewal in Female Germline Stem Cells [J]. Stem Cells. 2019;37(8):1095-107.

34. Gutierrez-Aguilar R, Thompson A, Marchand N, et al. The obesity-associated transcription factor ETV5 modulates circulating glucocorticoids [J]. Physiol Behav. 2015;150:38-42.

35. Gutierrez-Aguilar R, Kim DH, Casimir M, et al. The role of the transcription factor ETV5 in insulin exocytosis [J]. Diabetologia. 2014;57(2):383-91.

36. Puli OR, Danysh BP, McBeath E, et al. The Transcription Factor ETV5 Mediates BRAFV600E-Induced Proliferation and TWIST1 Expression in Papillary Thyroid Cancer Cells [J]. Neoplasia. 2018;20(11):1121-34.

\section{Figures}




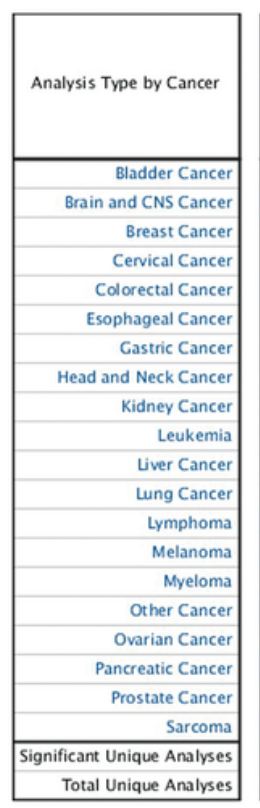

ETV1 ETV4 ETV5

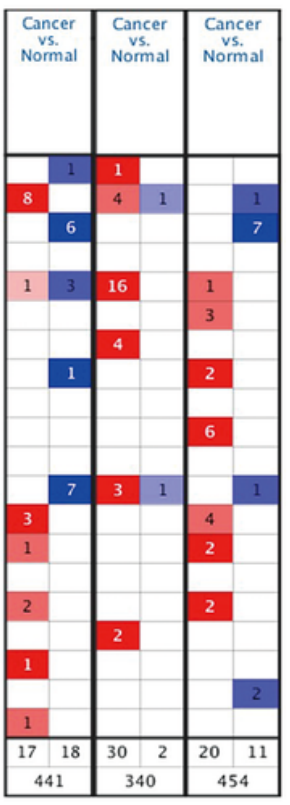

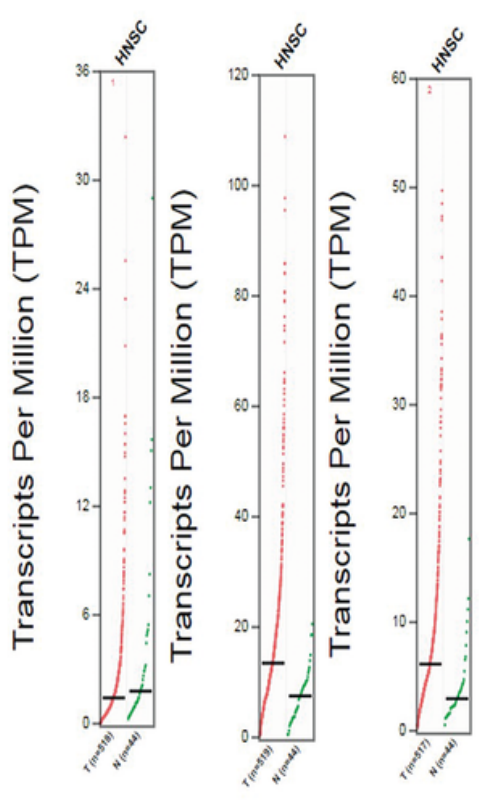

C
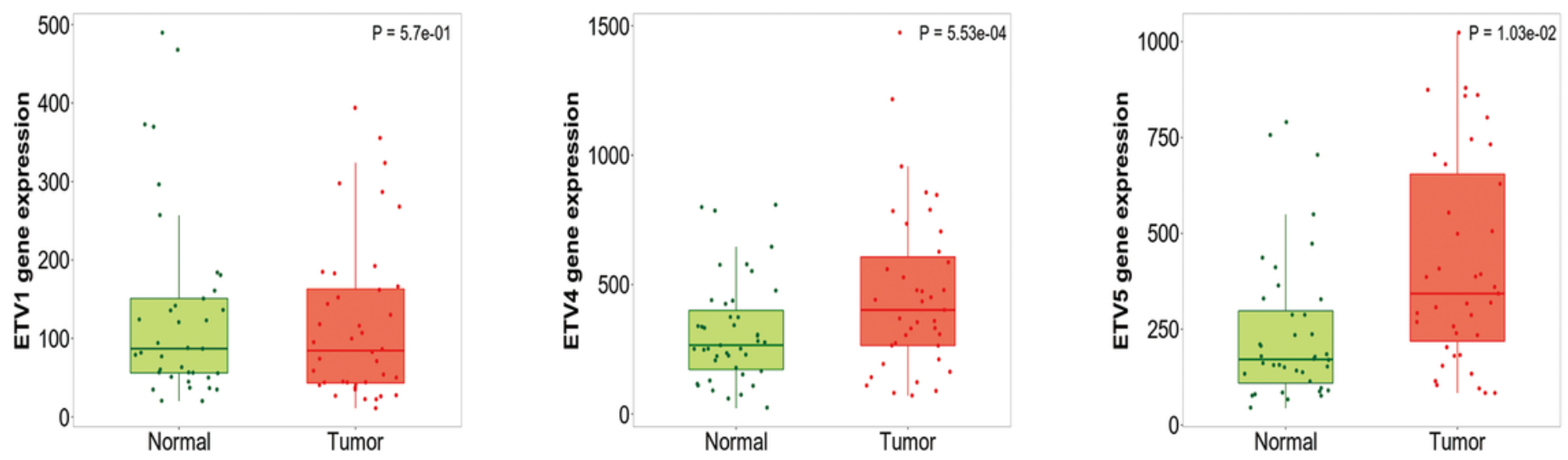

Figure 1

A. Transcriptional levels of PEA3 subfamily in cancers and standard samples (ONCOMINE database). B. Expression of PEA3 subfamily in HNSCC (GEPIA). C. Box plot expression of PEA3 subfamily in HNSCC (TNM plot). 

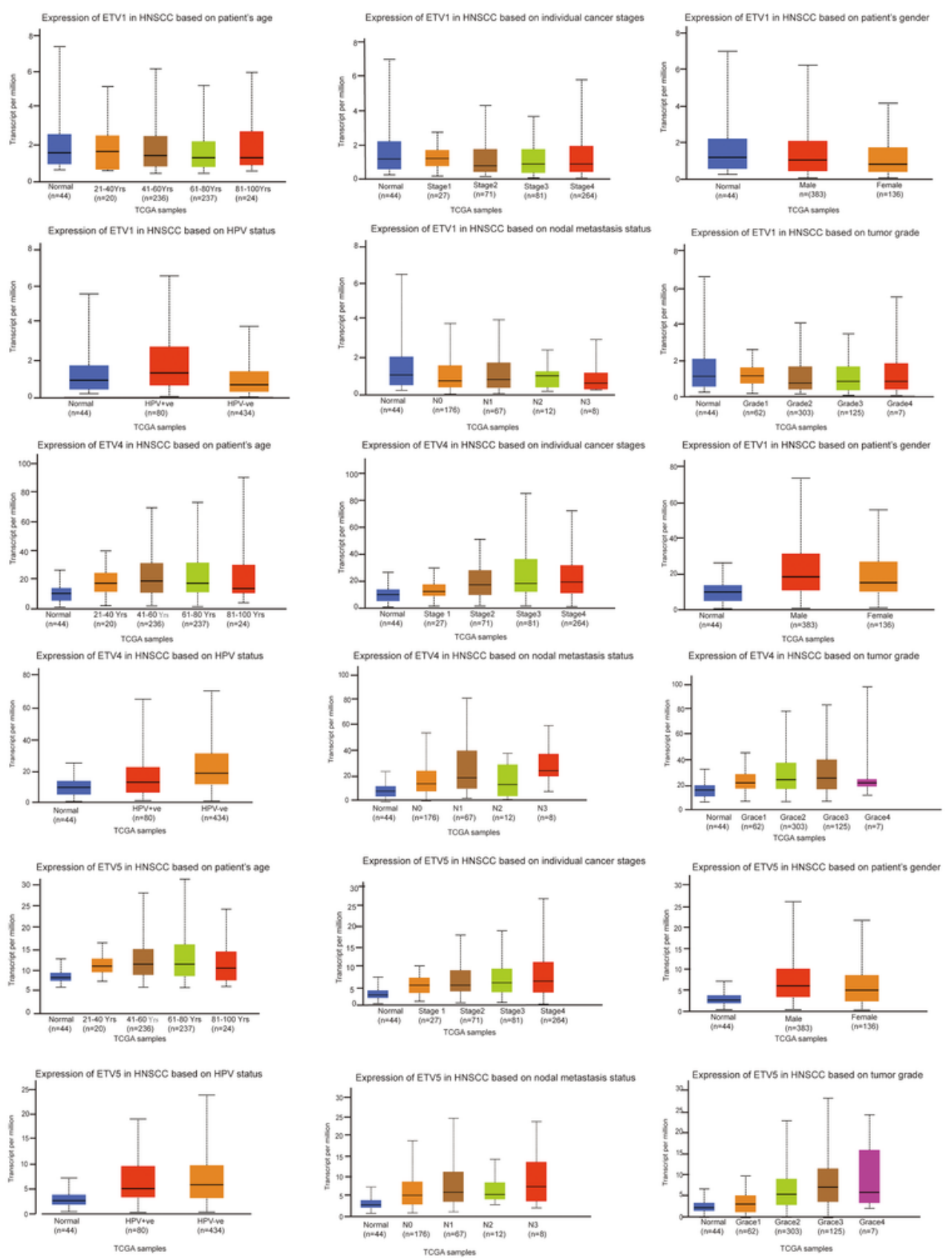

\section{Figure 2}

Boxplot showing the relative expression of PEA3 subfamily in subgroups of patients with HNSCC and those stratified based on gender, age, HPV status, cancer, stage, nodal metastasis status, and tumor grade (UALCAN). 

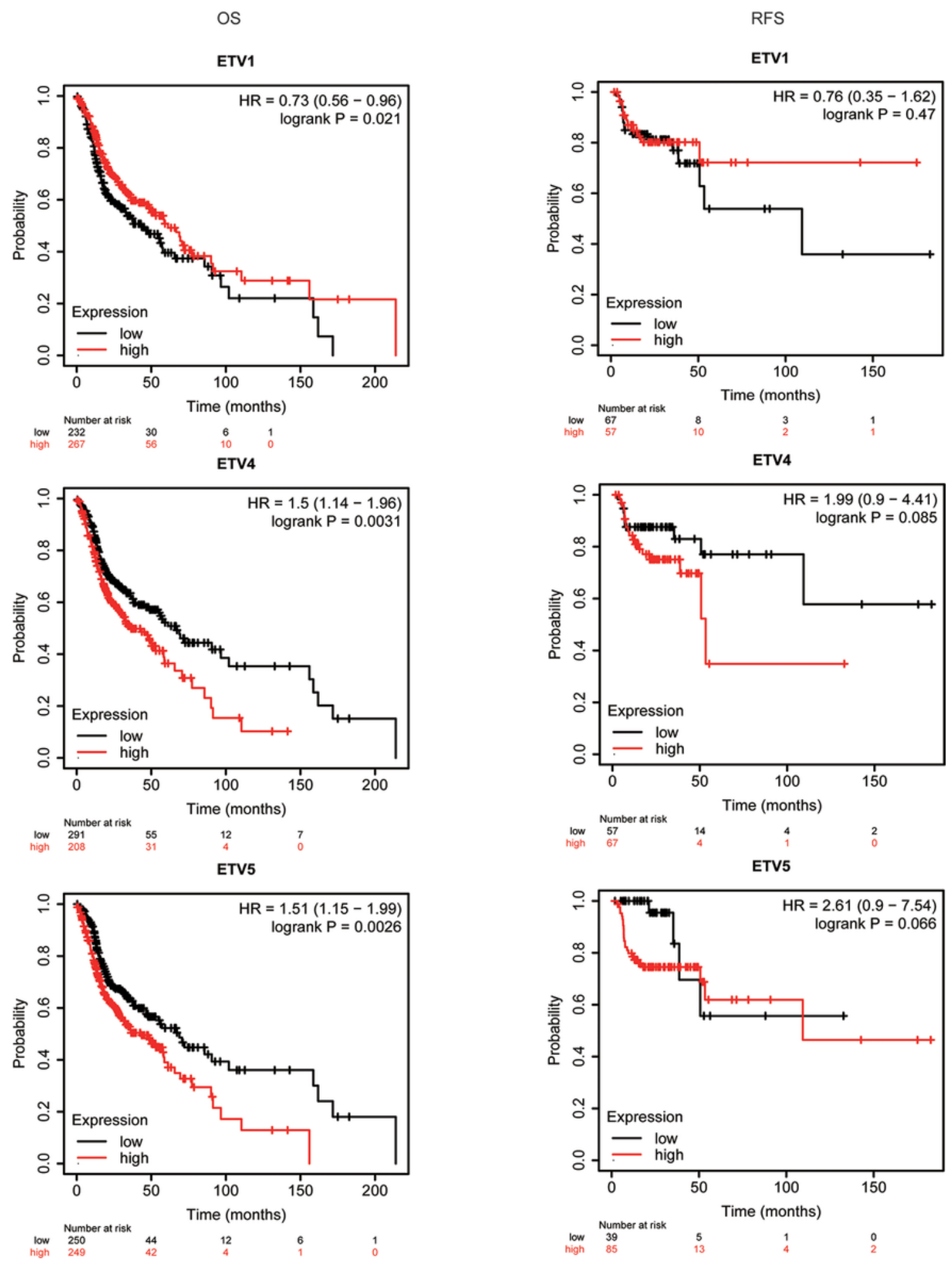

Figure 3

Prognostic value of the mRNA level of PEA3 subfamily in patients with HNSCC (Kaplan-Meier plotter database). 
A

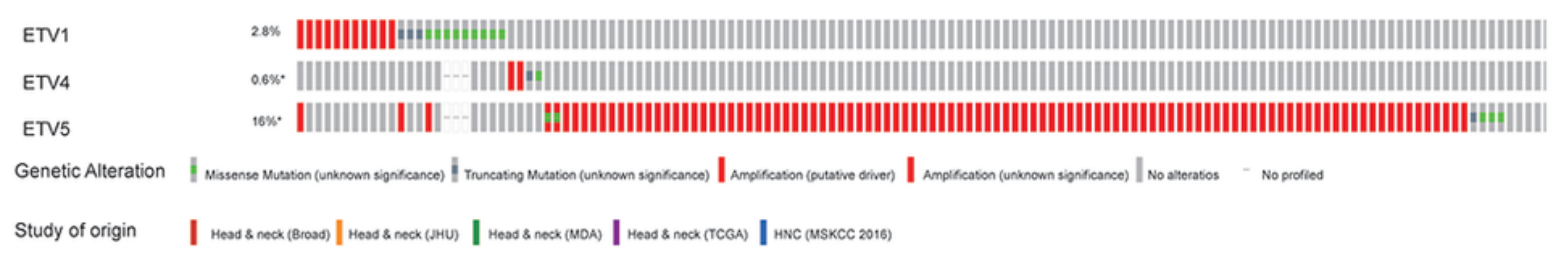

B

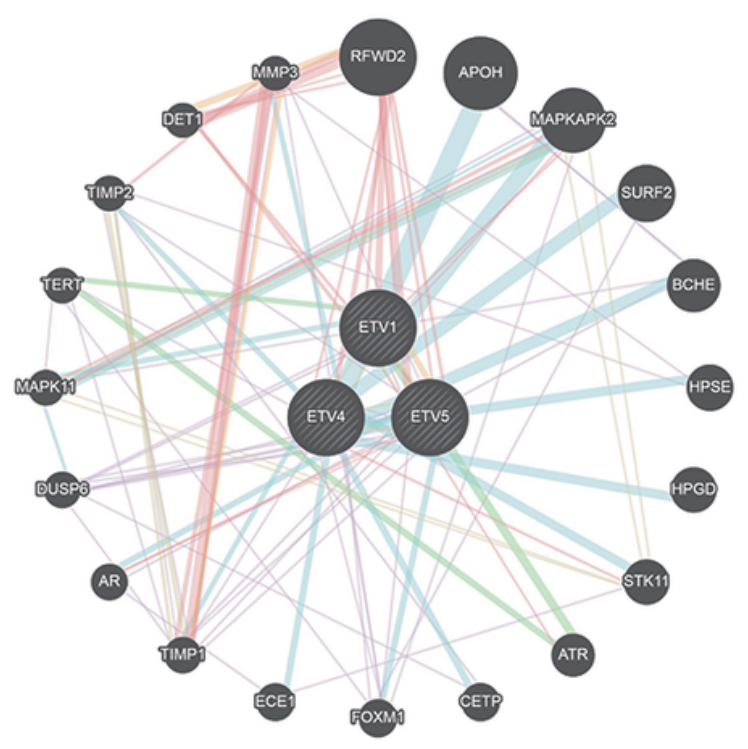

Figure 4

PEA3 expression and mutation analysis in HNSCC. A. PEA3 subfamily expression and mutation analysis (cBioPortal database). B. Network for PEA3 and 22 most frequently altered neighboring genes (GeneMANIA). 
A

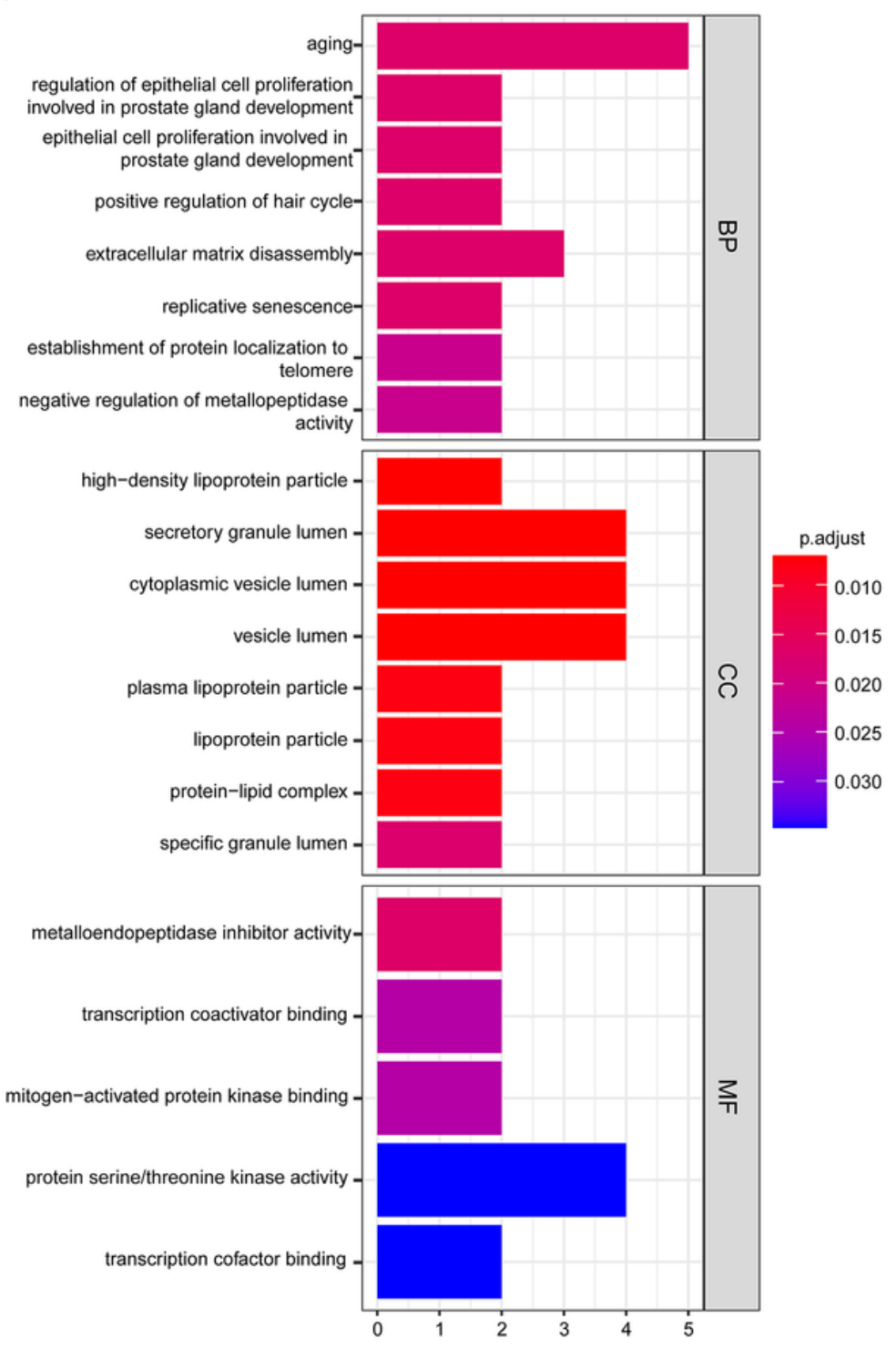

B

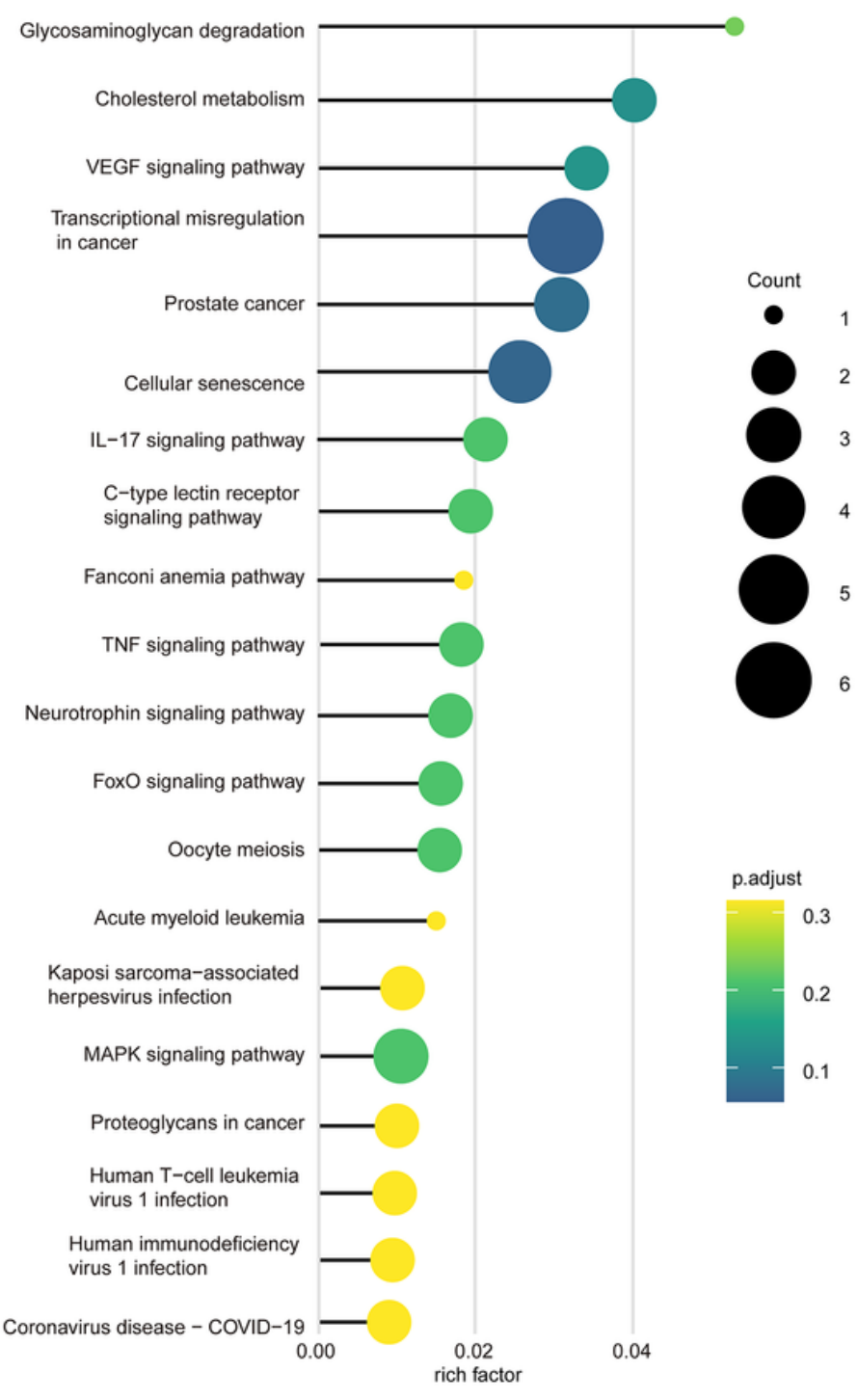

Figure 5

Predicted functions and pathways of PEA3 subfamily and their frequently altered neighboring genes in patients with HNSCC. GO enrichment analysis predicted the functional roles of target host genes based on BP, CC, and MF. KEGG pathway analysis identified the enriched pathways. BP: biological process; CC: cellular component; GO: Gene Ontology; KEGG: Kyoto Encyclopedia of Genes and Genomes; MF: molecular function. 


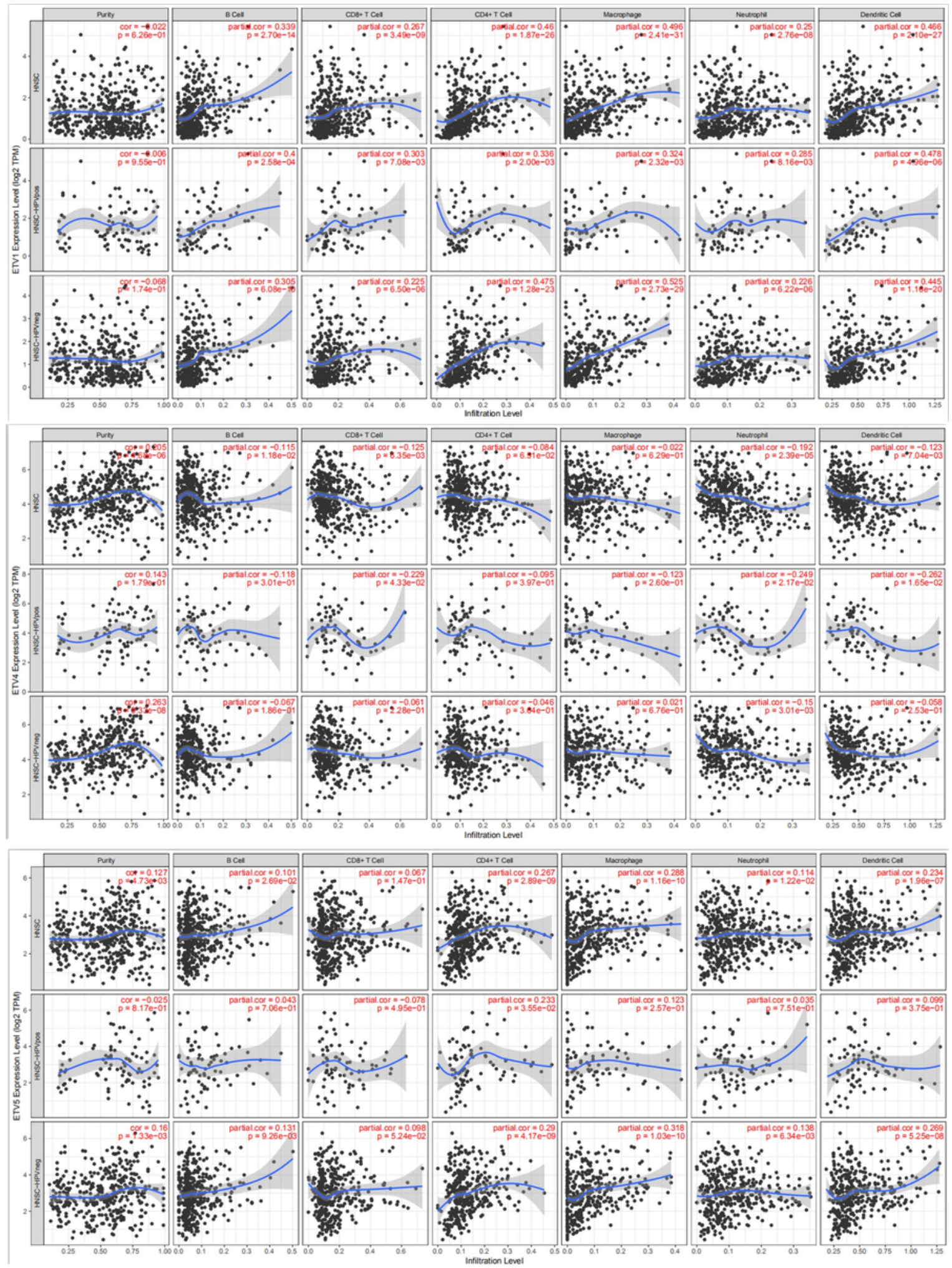

Figure 6

Correlation between PEA3 subfamily in HNSCC, HNSCC-HPV-pos, and HNSCC-HPV-neg expression. The abundance of immune infiltrates was statistically significant ( $p<0.05$, TIMER database). 


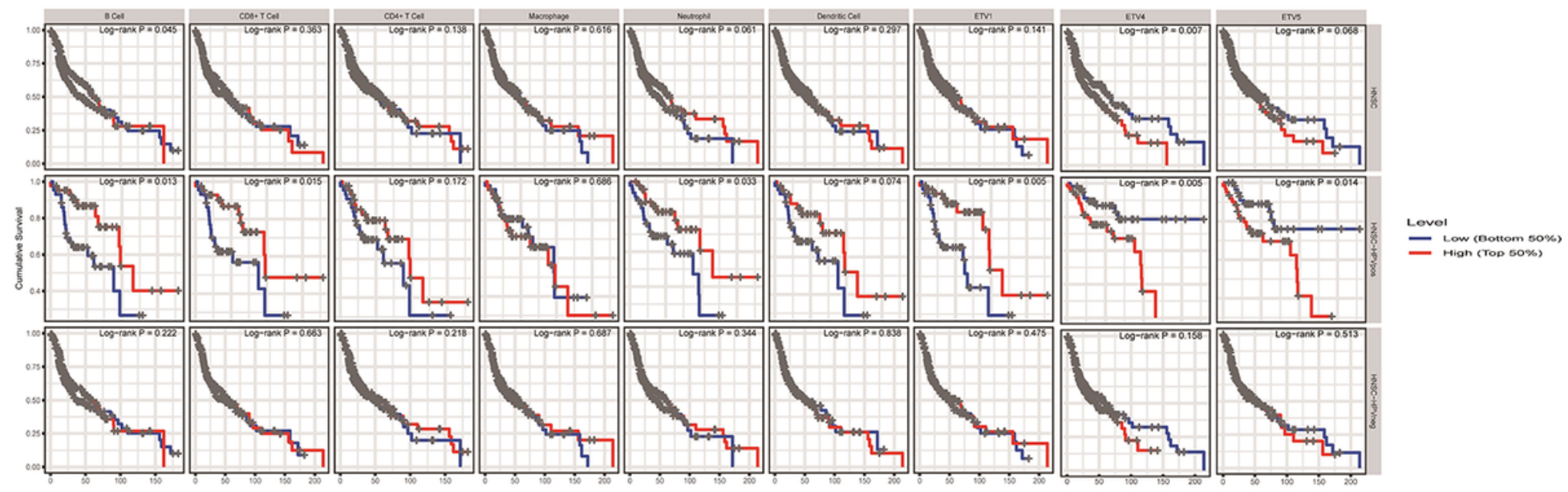

Figure 7

The difference in cumulative survival between HNSCC, HNSCC-HPV-pos, and HNSCC-HPV-neg. HNSCCHPV-pos group showed statistically significant immune infiltrates in the B cells, CD8+ T cells, and neutrophils $(p<0.05)$ of the PEA3 subfamily. ( TIMER database) 